PROCEEDINGS OF THE AMERICAN MATHEMATICAL SOCIETY

Volume 124, Number 8, August 1996

\title{
ON THE PRIME MODEL PROPERTY
}

\author{
LUDOMIR NEWELSKI
}

(Communicated by Andreas R. Blass)

\begin{abstract}
Assume $T$ is superstable, $\Phi(x)$ is a formula over $\emptyset, Q=\Phi\left(M^{*}\right)$ is countable and $K_{Q}=\{M: M$ is countable and $\Phi(M)=Q\}$. We investigate models in $K_{Q}$ assuming $K_{Q}$ has the prime model property. We prove some corollaries on the number of models in $K_{Q}$. We show an example of an $\omega$-stable $T$ and $Q$ with $K_{Q}$ having exactly 3 models.
\end{abstract}

First we fix the general set-up. Throughout, $T$ is a countable complete theory in a first-order language $L, \Phi(x)$ is a formula of $L$ without parameters, $M^{*}$ is a countable model of $T$ and $Q=\Phi\left(M^{*}\right)$. We work within a monster model $\mathcal{C}=\mathcal{C}^{e q}$ of $T$. All models of $T$ we consider are elementary submodels of $\mathcal{C}$. Let $K_{Q}=\{M: M$ is countable and $\Phi(M)=Q\}$ and let $I\left(K_{Q}\right)$ be the number of models in $K_{Q}$, up to isomorphism.

The goal of this note is to develop some model theory of $K_{Q}$. As I pointed out in [Ne1, p.651], in general $K_{Q}$ can not be treated with all common model-theoretic tools, since for example $K_{Q}$ does not necessarily have the joint embedding property. It turns out however that if we assume just that $K_{Q}$ has the prime model property (defined below), then it is much more manageable, at least for stable $T$. $K_{Q}$ really may differ from an elementary class: I have found an example of $K_{Q}$ with $I\left(K_{Q}\right)=2$, while a theorem of Vaught [Sa] says that $I\left(T, \aleph_{0}\right) \neq 2$. In this example $T$ is weakly minimal, but $K_{Q}$ does not have the prime model property. In this paper we give an example of an $\omega$-stable $T$ with $I\left(T, \aleph_{0}\right)=\aleph_{0}$ and a strongly minimal $Q$ with $I\left(K_{Q}\right)=3$.

Now we recall some basic notions from [Ne1]. We call $f \in A u t(\mathcal{C})$ a $Q$-mapping if $f[Q]=Q$. For $a \subset M \in K_{Q}$ let $A u t_{Q}(\mathcal{C} / a)$ be the group of $Q$-mappings fixing $a$ pointwise. This group acts in a Borel way on $S(Q a)$, inducing equivalence relation $E(a)$ on $S(Q a)$. The orbits of this action, that is the $E(a)$-classes, are called pseudotypes over $Q a$. When $a=\emptyset$, we use $E$ to denote $E(a)$. Pseudotypes are Borel subsets of $S(Q a)$, and $E(a)$ is analytic. We say that $p \in S(Q a)$ is $Q$-isolated over $a$ if $p / E(a)$ is not meager. $p / E(a)$ is the pseudotype of $p$ over $Q a$ (that is the equivalence class as a subset of $S(Q a)$, not a quotient of some sort). $Q$-isolation has many nice properties (see [Ne1, Ne2]), for instance if $Q$-isolated types are dense in $S(Q)$ then there is $M \in K_{Q}$ such that each $b \subset M$ satisfies a $Q$-isolated type over $Q$. Such an $M$ is called $Q$-atomic. A $Q$-atomic model is unique up to isomorphism. Also, $t p(a b / Q)$ is $Q$-isolated iff $t p(a / Q)$ is $Q$-isolated and $t p(b / Q a)$ is $Q$-isolated over $a$.

Received by the editors August 26, 1994 and, in revised form, February 13, 1995.

1991 Mathematics Subject Classification. Primary 03C15, 03C45.

(C)1996 American Mathematical Society 
Let $\mathcal{B}(Q, a)=\left\{p \in S(Q a)\right.$ : there is no $M \in K_{Q}$ containing a realization of $\left.p\right\}$. $\mathcal{B}(Q, a)$ is a meager $F_{\sigma}$-subset of $S(Q a) . \mathcal{B}(Q)$ denotes $\mathcal{B}(Q, \emptyset)$. We think of the types in $\mathcal{B}(Q)$ as 'bad' types, since they can not be used to build models in $K_{Q}$. Types in $S(Q) \backslash \mathcal{B}(Q)$ are called good.

We say that $K_{Q}$ has the prime model property if the following holds.

(PM) For every $a$ with $t p(a / Q)$ good there is a model $M \in K_{Q}$ containing $a$, such that for every $M^{\prime} \in K_{Q}$ containing $a$, some $Q$-mapping fixing $a$ embeds $M$ into $M^{\prime}$.

The model $M$ occurring in (PM) is called $Q$-prime over $a$. ' $Q$-prime' means $Q$-prime over $\emptyset$.

Remark. (1) If $M$ is $Q$-prime over $a$ then $M$ is $Q$-atomic over $a$. In particular, a $Q$-prime over $a$ model is unique up to isomorphism over $a$. (However, even a countable $Q$-atomic model need not be $Q$-prime.)

(2) If (PM) holds and $p \in S(Q a)$ then $p$ is $Q$-isolated over $a$ iff every $M \in K_{Q}$ containing $a$ contains a realization of a type $p^{\prime} \in p / E(a)$.

(3) (PM) implies that $Q$-isolated over $a$ types are dense in $S(Q a)$ and " $Q$-prime over $a "=$ "Q-atomic over $a "$.

Proof. (1) Wlog $a=\emptyset$. We must show that if $p \in S(Q)$ is not $Q$-isolated then no $p^{\prime} \in p / E$ is realized in $M$. To prove this it is enough to show that some $N \in K_{Q}$ omits every $p^{\prime} \in p / E$. Since $p$ is not $Q$-isolated, $p / E$ is meager, hence is covered by countably many closed nowhere dense subsets $X_{n}, n<\omega$, of $S(Q)$. A closed subset $X_{n}$ of $S(Q)$ corresponds to a type $q_{n}$ over $Q$, and $X_{n}$ being nowhere dense means that $q_{n}$ is non-isolated. Hence by the omitting types theorem there is a model $N \in K_{Q}$ omitting every $q_{n}, n<\omega$. This means that $N$ omits every $p^{\prime} \in p / E$. (After all, the omitting types theorem is just the Baire category theorem adapted for the needs of model theorists.) Since a $Q$-atomic model is unique up to isomorphism, also a $Q$-prime model is such.

(2),(3) follow easily.

We say that $K_{Q}$ has the joint embedding property if the following holds.

(JE) For every $a$ with $t p(a / Q)$ good, if $M_{0}, M_{1} \in K_{Q}$ contain $a$ then for some $M \in K_{Q}$ containing $a$ there are $Q$-mappings $f_{0}, f_{1}$ fixing $a$ and embedding $M_{0}, M_{1}$ respectively into $M$.

We say that $M \in K_{Q}$ is $Q$-saturated if for every $a \subset M$ and good $p \in S(Q a)$, some type in $p / E(a)$ is realized in $M . M \in K_{Q}$ is $Q$-universal if for every $N \in K_{Q}$ there is a $Q$-mapping embedding $N$ into $M$.

Lemma 1. (1) If $T$ is stable, there are countably many good pseudotypes over $Q$ and $(P M)$ holds, then there is a $Q$-saturated model.

(2) A $Q$-saturated model is $Q$-universal and unique up to isomorphism.

(3) If ( $T$ is stable and (PM) holds) or (a Q-saturated model exists) then (JE) holds.

Proof. [Ne4, Corollary 2.10(3)] proves that if $T$ is stable and (PM) holds, then whenever the types $t p(a / Q), t p(b / Q)$ are good, then for some $b^{\prime}$ with $t p\left(b^{\prime} / Q\right) E t p(b / Q)$, the type $t p\left(a b^{\prime} / Q\right)$ is good. This amalgamation property easily implies the lemma.

The following fact ([Ne4, Corollary 2.10]) shows that the assumption of (PM) makes sense. 
Fact. Assume $T$ is small, stable, $T\lceil\Phi$ is $\omega$-stable and one of the following conditions holds.

(i) $T\left\lceil\Phi\right.$ is bounded, 1-based or of finite rank, and $I\left(T\left\lceil\Phi, \aleph_{0}\right)<2^{\aleph_{0}}\right.$.

(ii) $Q$ is atomic or saturated (as a model of $T\lceil\Phi$ ).

(iii) $T$ is superstable and $T\left\lceil\Phi\right.$ has $<2^{\aleph_{0}}$ countable models.

Then $K_{Q}$ has the prime model property and there are countably many good pseudotypes over $Q$.

It would be very good if we could omit the assumption in (i) that $T\lceil\Phi$ is bounded, 1-based or of finite rank. Then we could drop (iii) in the Fact. It is open whether $T$ stable, $T\left\lceil\Phi \omega\right.$-stable and $I\left(T, \aleph_{0}\right)<2^{\aleph_{0}}$ implies $(\mathrm{PM})$. This is related to the $\tau$-stability conjecture $[\mathrm{Ne} 2, \mathrm{Ne} 4]$. As I mentioned above, there is an example of a weakly minimal $T$ and $K_{Q}$ with $I\left(K_{Q}\right)=2$. In this example $K_{Q}$ does not have the prime model property. Now we give an example of an $\omega$-stable $T$ with $I\left(T, \aleph_{0}\right)=\aleph_{0}$ and a strongly minimal $Q$ with $I\left(K_{Q}\right)=3$.

Example. The example is a modification of Shelah's example of a theory $T_{0}$ with abnormal types ([Ba, XVIII,4]). Let $V$ be a countably infinite set and $P=\left\{c_{n}, n<\right.$ $\omega\}$ an infinite and co-infinite subset of $V$. Equip $V \backslash P$ with a structure of a model of $T_{0}$. This means among others that there is an equivalence relation $E^{\prime}$ on $V \backslash P$ and an asymmetric relation $R$ on $(V \backslash P) / E^{\prime}$ such that $\left((V \backslash P) / E^{\prime} ; R\right)$ is a directed graph without cycles such that each element has infinitely many successors and predecessors (see $[\mathrm{Ba}]$ for details). We can arrange that $\left((V \backslash P) / E^{\prime} ; R\right)$ is connected. So on $(V \backslash P) / E^{\prime}$ there is a natural distance function $d(x, y)$. Define a ternary relation $S \subset P \times(V \backslash P) / E^{\prime} \times(V \backslash P) / E^{\prime}$ by: $S\left(c_{n}, x, y\right)$ iff $d(x, y) \leq n$.

Let $M^{*}=\left(V ; P, S, c_{n}, n<\omega\right.$; the structure of $V \backslash P$ as a model of $\left.T_{0}\right)$, and $T=T h\left(M^{*}\right)$. Clearly, $T$ is $\omega$-stable, $I\left(T, \aleph_{0}\right)=\aleph_{0}$, and $\Phi(x)=P(x)$ is trivial and strongly minimal. Let $M^{\prime}$ be a prime model of $T$ and $Q=P\left(M^{\prime}\right)$. Then for any $M \in K_{Q}, \neg P(M) / E$ is $R$-connected. By [Ba, XVIII,4.6] in $T_{0}$, up to isomorphism there are 3 kinds of countable connected components. Hence $I\left(K_{Q}\right)=3$. $\omega$ stability implies $K_{Q}$ has the prime model property, hence by Theorem 1 below we can not have here $I\left(K_{Q}\right)=2$. However, if $M$ is a countable model of $T$ with $P(M) \neq\left\{c_{n}, n<\omega\right\}$, then for $Q=\Phi(M), I\left(K_{Q}\right)$ is infinite.

In this paper we try to generalize a result of Lachlan [Ba] saying that for superstable $T, I\left(T, \aleph_{0}\right)>1$ implies $I\left(T, \aleph_{0}\right) \geq \aleph_{0}$. We shall use the following lemma.

Lemma 2 ([Ne1, Lemma 2.4]). If $T$ is stable, $t p(a / Q b)$ is $Q$-isolated over $b$ and $t p(a / Q)$ is not $Q$-isolated, then a $\backslash b(Q)$.

We write $a \downarrow b(X)$ for ' $a$ is independent from $b$ over $X$ '. Regarding the theorem of Vaught we can prove the following.

Theorem 1. If $T$ is superstable and $K_{Q}$ has the prime model property, then $I\left(K_{Q}\right)$ $\neq 2$. If moreover $T$ has finite $U$-rank and $I\left(K_{Q}\right)>1$, then $I\left(K_{Q}\right)$ is infinite.

Proof. Suppose $I\left(K_{Q}\right)>1$. If every good type in $S_{n}(Q), n<\omega$, is $Q$-isolated, then every model in $K_{Q}$ is $Q$-atomic, hence $I\left(K_{Q}\right)=1$, a contradiction. So let $p \in S(Q) \backslash \mathcal{B}(Q)$ be non- $Q$-isolated. Let $M_{0}$ be the $Q$-prime model.

If $\left(S_{n}(Q) \backslash \mathcal{B}(Q)\right) / E$ is uncountable, then clearly $I\left(K_{Q}\right)$ is infinite. So we can assume there are only countably many good pseudotypes over $Q$. By Lemma 1(1) we see that there is a $Q$-saturated model $M_{\omega}$. Since $M_{\omega}$ realizes a non- $Q$-isolated type, $M_{0} \neq M_{\omega}$. 
Let $a$ realize $p$ and let $M$ be $Q$-prime over $a$. Wlog $M_{0} \subseteq M$. Since $p$ is not $Q$-isolated, $a \in M \backslash M_{0}$. Since $Q=\Phi(M)=\Phi\left(M_{0}\right), M$ and $M_{0}$ are a Vaughtian pair. In particular, $t p\left(a / M_{0}\right)$ is non-algebraic and orthogonal to $\Phi$. Choose a finite $b \subset M_{0}$ with $a \downarrow M_{0}(b)$. So also $t p(a / Q b) \perp \Phi$. Also, $t p(a / Q b)$ is not $Q$-isolated over $b$. Otherwise, since $t p(b / Q)$ is $Q$-isolated (as $b \subset M_{0}$ ), by transitivity of $Q$-isolation ([Ne1]) we would get that $p=\operatorname{tp}(a / Q)$ is $Q$-isolated.

Let $I=\left\{a_{n}, n<\omega\right\}$ be a Morley sequence in $\operatorname{stp}(a / Q b)$, with $a=a_{0}$. Wlog $I \subseteq$ $M_{\omega}$. By superstability, for some $n^{\prime}$, if $n>n^{\prime}$ then $a_{n} \downarrow Q b\left(a_{<n}\right)$ and $t p\left(a_{n} / a_{<n}\right)$ is stationary. Hence for $n>n^{\prime}$ also $t p\left(a_{n} / Q b a_{<n}\right)$ is not $Q$-isolated over $b a_{<n}$. Indeed, since $t p\left(a_{n} / b a_{<n}\right)$ is orthogonal to $\Phi$, by the open mapping theorem it is enough to show that $t p\left(a_{n} / b a_{<n}\right)$ is non-isolated. Suppose otherwise. Since $a \downarrow M_{0}(b)$ the construction yields $a_{n} \downarrow a_{<n}(b)$. This gives that $t p\left(a_{n} / b\right)=t p(a / b)$ is isolated and stationary, because $t p\left(a_{n} / b a_{<n}\right)$ is stationary. Hence $t p(a / b) \vdash t p(a / Q b)$, and $t p(a / Q b)$ is isolated, hence $Q$-isolated over $b$, a contradiction.

The rest of the proof resembles that of [Ne1, Theorem 2.5]. Let $M_{n}$ be $Q$-prime over $b a_{<n}$. Clearly for $n>0, M_{n} \neq M_{0}$, since $p$ is realized in $M_{n}$. Let $m>n^{\prime}$ and suppose $M_{m}$ and $M_{\omega}$ are isomorphic. Then within $M_{m}$ there is a copy $b^{\prime}\left\{a_{n}^{\prime}, n<\right.$ $\omega\}$ of $b I$ (via some $Q$-mapping). By the properties of $Q$-isolation, for every $c \subset$ $M_{m}, M_{m}$ is $Q$-atomic over $b a_{<m} c$. Hence $M_{m}$ is $Q$-atomic over $b a_{<m} b^{\prime} a_{<n}^{\prime}$ for any $n$. In particular, for every $n>n^{\prime}$ we have that $t p\left(a_{n}^{\prime} / Q b a_{<m} b^{\prime} a_{<n}^{\prime}\right)$ is $Q$-isolated over $b a_{<m} b^{\prime} a_{<n}^{\prime}$ and $t p\left(a_{n}^{\prime} / Q b^{\prime} a_{<n}^{\prime}\right)$ is not $Q$-isolated over $b^{\prime} a_{<n}^{\prime}$. By Lemma 2 we get that for every $n>n^{\prime}, b a_{<m}\left\lfloor a_{n}^{\prime}\left(Q b^{\prime} a_{<n}^{\prime}\right)\right.$, contradicting the superstability of $T$.

The case when $U$-rank is finite is handled like in [Ne1]. We prove that if $k$ is large enough then $M_{k} \neq M_{m}$.

I managed to generalize Lachlan's theorem for $K_{Q}$ in case when $Q$ is weakly saturated (as a model of $T\lceil\Phi$ ), that is when every type in $T\lceil\Phi$ is realized in $Q$.

Theorem 2. If $T$ is superstable, $K_{Q}$ has the prime model property, $Q$ is weakly saturated and $I\left(K_{Q}\right)>1$, then $I\left(K_{Q}\right)$ is infinite.

The proof relies on the following lemma.

Lemma 3. If $T$ is superstable and $Q$ is weakly saturated, then every type in $S(\emptyset)$ has a good extension in $S(Q)$.

Proof. Choose any $a$. We want to find $a^{\prime} \equiv a$ with $t p\left(a^{\prime} / Q\right)$ good. By superstability, for some $b \subset \Phi(\mathcal{C})$ we have $a \downarrow \Phi(\mathcal{C})(b)$. This implies that if $b \subset Q^{\prime}=\Phi\left(M^{\prime}\right)$ for some countable $M^{\prime}$, then $t p\left(a / Q^{\prime}\right) \notin \mathcal{B}\left(Q^{\prime}\right)$. Indeed, otherwise for some $d \in \Phi(\mathcal{C})$ we would have $a\left\lfloor d\left(Q^{\prime}\right)\right.$, while $a \downarrow \Phi(\mathcal{C})\left(Q^{\prime}\right)$, a contradiction.

Since $Q$ is weakly saturated, there is $b^{\prime} \subset Q$ with $b \equiv b^{\prime}$. Choose $a^{\prime}$ with $a^{\prime} b^{\prime} \equiv a b$. It follows that $a^{\prime} \downarrow \Phi(\mathcal{C})\left(b^{\prime}\right)$ and $t p\left(a^{\prime} / Q\right)$ is good.

Note that if in Lemma 3 we weaken the assumption of superstability to stability, then we must assume that $Q$ is universal.

Proof of Theorem 2. As in the proof of Theorem 1 we find $a, b$ with $t p(a / Q b)$ non$Q$-isolated over $b$ and orthogonal to $\Phi$ (hence good). We can also assume that $a \downarrow Q(b)$ and $t p(a / b)$ is stationary non-isolated (see the proof of Theorem 1). Let $k=w(a b)$. 
By Lemma 3, for each $n<\omega$ there is a Morley sequence $A_{n}$ in $\operatorname{stp}(a b)$ of length $\left(k^{n+1}-1\right) /(k-1)$ such that $t p\left(A_{n} / Q\right)$ is good. Let $M_{n}$ be $Q$-prime over $A_{n}$. Let $\left|A_{n}\right|$ denote the length of $A_{n}$ and let $w\left(A_{n}\right)$ be the weight of $A_{n}$. Notice that

$$
w\left(A_{n}\right)=k \frac{k^{n+1}-1}{k-1}=\left|A_{n+1}\right|-1
$$

Hence for $n<m, w\left(A_{n}\right)<\left|A_{m}\right|$.

We shall prove that for $n<m, M_{n}$ and $M_{m}$ are non-isomorphic. Suppose not. Then there is an $A_{m}^{\prime} \subset M_{n}$, a Morley sequence in $\operatorname{stp}(a b)$ of length $\left|A_{m}\right|$. Since $w\left(A_{n}\right)<\left|A_{m}\right|$, for some $a^{\prime} b^{\prime} \in A_{m}^{\prime}, a^{\prime} b^{\prime} \downarrow A_{n}$. In particular, $a^{\prime} \downarrow A_{n}\left(b^{\prime}\right)$. Since $\operatorname{tp}\left(a^{\prime} / b^{\prime}\right)$ is orthogonal to $\Phi$, also $a^{\prime} \smile A_{n} Q\left(b^{\prime}\right)$. As $M_{n}$ is $Q$-atomic over $A_{n}, M_{n}$ is also $Q$-atomic over $A_{n} b^{\prime}$. Hence $t p\left(a^{\prime} / A_{n} Q b^{\prime}\right)$ is $Q$-isolated over $A_{n} b^{\prime}$. As $t p\left(a^{\prime} / b^{\prime}\right)$ is stationary, non-isolated and orthogonal to $\Phi, t p\left(a^{\prime} / Q b^{\prime}\right)$ is not $Q$-isolated over $b^{\prime}$. By Lemma 2, $a^{\prime}\left\lfloor A_{n}\left(Q b^{\prime}\right)\right.$, a contradiction.

Corollary 1. Assume $T$ is superstable, $T\lceil\Phi$ is $\omega$-stable and $Q$ is saturated. Then $I\left(K_{Q}\right)>1$ implies $I\left(K_{Q}\right)$ is infinite. On the other hand, $I\left(K_{Q}\right)=1$ implies $T$ is $\omega$-stable and $(\omega, \omega)$-categorical relative to $\Phi$ (in the sense of $[\mathrm{HHM}]$ ).

Proof. If $T$ is not small, then Lemma 3 implies that $I\left(K_{Q}\right)$ is infinite. So assume $T$ is small. By the Fact, $K_{Q}$ has the prime model property. Hence by Theorem 2 , $I\left(K_{Q}\right)>1$ implies $I\left(K_{Q}\right)$ is infinite.

Now assume $I\left(K_{Q}\right)=1$. If $T$ is not $\omega$-stable then for some $a$ there is an isolated type $p \in S(a)$ without Morley rank, such that each type of smaller $\infty$-rank has Morley rank (see e.g. [Ne3]). It follows that $p$ has infinite multiplicity, hence if $b$ realizes $p$ then $p^{\prime}=p \mid a b$ is non-isolated. By Lemma 3, we can assume that $t p(a b / Q)$ is good. Since $p^{\prime} \perp \Phi$, we have $p^{\prime} \vdash p^{\prime \prime}$ for some $p^{\prime \prime} \in S(Q a b)$. Clearly $p^{\prime \prime}$ is not $Q$-isolated over $a b$. Hence $I\left(K_{Q}\right)>1$, a contradiction.

So $T$ is $\omega$-stable. It follows that the only model in $K_{Q}$ is prime over $Q$ in the usual sense.

Now suppose $Q^{\prime}=\Phi\left(M^{\prime}\right)$ is countable. Wlog $Q^{\prime} \subseteq Q$. If some $p \in S\left(Q^{\prime}\right)$ is non-isolated and good then also $p^{\prime}=p \mid Q$ is non-isolated and good. $p^{\prime}$ is realized in a model in $K_{Q}$ which is not prime over $Q$, a contradiction. We see that every model in $K_{Q^{\prime}}$ is prime over $Q^{\prime}$. Hence $T$ is $(\omega, \omega)$-categorical relative to $\Phi$ in the sense of $[\mathrm{HHM}]$.

I would like to stress the fact, implicit in the proof of Theorem 2, that the more ample $Q$ is, the larger $I\left(K_{Q}\right)$ is. To make this more explicit, we prove the following corollary, which deals with the case of strongly minimal $\Phi$, when we have a good notion of dimension of $Q$.

Corollary 2. Assume $T$ is small superstable, $\Phi$ is strongly minimal and $I\left(K_{Q}\right)>$ 1. Then there are $k, l<\omega$ such that if $Q^{\prime}=\Phi\left(M^{\prime}\right)$ is countable, $n>2$ and

$$
\operatorname{dim}\left(Q^{\prime}\right) \geq \frac{k^{n}-k}{k-1}+l
$$

then $I\left(K_{Q^{\prime}}\right) \geq n$.

Proof. By the Fact, $K_{Q^{\prime}}$ has the prime model property. Also we can assume that there are countably many good pseudotypes over $Q^{\prime}$, hence there is a $Q^{\prime}$-saturated 
model. Wlog the strongly minimal type $r \in S(\emptyset)$ containing $\Phi$ is eventually nonisolated. Let $l$ be the largest number such that $r^{l}$ is isolated. First we prove the following variant of Lemma 3 .

(a) If $p \in S(\emptyset)$ and $\operatorname{dim}\left(Q^{\prime}\right) \geq w(p)+l$ then there is $p^{\prime} \in S\left(Q^{\prime}\right) \backslash \mathcal{B}\left(Q^{\prime}\right)$ extending $p$.

Indeed, let $a$ realize $p$ and $C=C b(a / \Phi(\mathcal{C}))$. Since $C \subseteq a c l(a), w(C) \leq w(a)$. Choose a Morley sequence $I$ in $r$ of size $l$, with $I \downarrow C$, and let $Q^{\prime \prime}$ be a model of $T\left\lceil\Phi\right.$ prime over $C I$. Clearly $w(C / I)=w(C)$. If $d \in Q^{\prime \prime}$ realizes $r \mid I$ then $t p(d / C I)$ is isolated and $t p(d / I)$ is non-isolated, hence $d\left\lfloor C(I)\right.$. Thus $\operatorname{dim}\left(r \mid I, Q^{\prime \prime}\right) \leq w(C)$, and $\operatorname{dim}\left(Q^{\prime \prime}\right) \leq w(C)+l \leq \operatorname{dim}(Q)$. In particular, $Q^{\prime \prime}$ may be embedded into $Q^{\prime}$. Now (a) follows as in Lemma 3.

Now we find $a, b$ as in the proof of Theorem 2. Let $k=w(a b)$. Hence $w\left(A_{n}\right)=$ $\left(k^{n+2}-k\right) /(k-1)$. If

$$
\operatorname{dim}\left(Q^{\prime}\right) \geq \frac{k^{n+2}-k}{k-1}+l
$$

then by (a), for $m \leq n$ we can find $A_{m}^{\prime} \equiv A_{m}$ with $t p\left(A_{m}^{\prime} / Q^{\prime}\right)$ good.

Let $M_{0}$ be $Q^{\prime}$-prime, for $0<m \leq n$ let $M_{m}$ be $Q^{\prime}$-prime over $A_{m}^{\prime}$ and let $M_{n+1}$ be $Q^{\prime}$-saturated. The proof of Theorem 2 shows that $M_{m}, m \leq n$, are nonisomorphic. The proof of Theorem 1 shows they are also non-isomorphic to $M_{n+1}$. Hence $I\left(K_{Q^{\prime}}\right) \geq n+2$.

We showed above an example of an $\omega$-stable $T$ and $Q$ with $I\left(K_{Q}\right)=3$. It may be interesting to point that any such example should resemble the classical situation.

Proposition. Assume $T$ is superstable, $K_{Q}$ has the prime model property and $I\left(K_{Q}\right)=3$. Then the 3 models in $K_{Q}$ are: the $Q$-prime one, the $Q$-saturated one and the third model $M$ which is neither $Q$-prime nor $Q$-saturated. $M$ is characterized by the following condition.

(*) For every a with $t p(a / Q)$ good and not $Q$-isolated, $M$ is isomorphic to a model $Q$-prime over a.

Proof. First we prove that $M$ satisfies $(*)$. Suppose $t p(a / Q)$ is good and not $Q$ isolated, and let $N$ be $Q$-prime over $a$. So $N$ is not $Q$-prime. The proof of Theorem 1 shows $N$ is not $Q$-saturated. Hence $N$ is isomorphic to $M$. This shows (*). The other direction is equally easy.

Most of the results of this note may be adapted to the situation when $Q$ is fixed pointwise by $\operatorname{Aut}(\mathcal{C})$, that is when the elements of $Q$ are named by constants of the language.

\section{REFERENCES}

[Ba] J.T. Baldwin, Fundamentals of stability theory, Springer, 1987. MR 89k:03002

[HHM] W. Hodges, I.M. Hodkinson, D. Macpherson, Omega-categoricity, relative categoricity and coordinatization, Ann. Pure Appl. Logic 46(1990), 169-199. MR 91g:03066

[Ne1] L. Newelski, A model and its subset, J. Symb. Logic 57(1992), 644-658. MR 93h:03046

[Ne2] L. Newelski, Scott analysis of pseudo-types, J. Symb. Logic 58(1993), 648-663. MR 94m:03055 
[Ne3] L. Newelski, Meager forking, Ann.Pure Appl.Logic 70(1994), 141-175. MR 96a:03047

[Ne4] L. Newelski, On atomic or saturated sets, J. Symb. Logic, submitted.

[Sa] G. Sacks, Saturated Model Theory, Benjamin, Reading 1972. MR 53:2668

Mathematical Institute, Polish Academy of Sciences, ul.Kopernika 18, 51-617 WroCLAW, POLAND

Current address: Mathematical Institute, Wrocław University, pl. Grunwaldzki 2/4, 50-384 Wrocław, Poland

E-mail address: newelski@math.uni.wroc.pl 